Louisiana State University

LSU Digital Commons

Faculty Publications

Department of Physics \& Astronomy

6-25-2007

\title{
The expansion of the universe and the cosmological constant problem
}

R. F. O'Connell

Louisiana State University

Follow this and additional works at: https://digitalcommons.Isu.edu/physics_astronomy_pubs

\section{Recommended Citation}

O'Connell, R. (2007). The expansion of the universe and the cosmological constant problem. Physics Letters, Section A: General, Atomic and Solid State Physics, 366 (3), 177-178. https://doi.org/10.1016/ j.physleta.2007.02.006

This Article is brought to you for free and open access by the Department of Physics \& Astronomy at LSU Digital Commons. It has been accepted for inclusion in Faculty Publications by an authorized administrator of LSU Digital Commons. For more information, please contact ir@lsu.edu. 


\title{
The Expansion of the Universe and the Cosmological Constant Problem
}

\author{
R. F. O'Connell \\ Department of Physics and Astronomy, \\ Louisiana State University, Baton Rouge, LA 70803-4001
}

(Dated: November 18, 2018)

\begin{abstract}
The discovery that the expansion of the universe is accelerating in time is a major discovery which still awaits adequate explanation. It is generally agreed that this implies a cosmic repulsion as a result of the existence of a cosmological constant $\wedge>0$. However, estimates of $\wedge$, based on calculations of the zero-point fluctuations of quantum fields are too large by over a hundred orders of magnitude. This result is obtained by summing the zero-point energies up to a large cutoff energy $\Omega$, based on the Planck scale. Since there is no compelling reason for this choice, we argue that since all known quantum electrodynamic (QED) effects involves interaction with matter, a preferred choice should be based on causality and other considerations, leading to a much lower value for $\wedge$.
\end{abstract}


Measurements of the expansion of the universe [1] imply a vacuum energy density which is over a hundred orders of magnitude too small compared to current theoretical calculations [2, 3] which attribute this so-called dark energy to quantum zero-point energies. The latter embrace a variety of fields but the essence of our remarks here can be captured by concentrating on just the normal modes of the electromagnetic field. The vacuum energy density for the E-M fields is simply given by summing the zero-point energies $(\hbar \omega / 2)$ of all the normal modes, up to a cutoff frequency $\Omega$, to get (after inclusion of a factor 2 arising from the fact that there are two normal modes of the E-M field for each wave vector)

$$
<\rho>=2 \int_{0}^{p_{\max }} d p \frac{4 \pi p^{2}}{(2 \pi \hbar)^{3}} \frac{\hbar \omega}{2}
$$

where $p_{\max }$ is the momentum cutoff. Hence, since $p=(\hbar \omega / c)$, we obtain,

$$
<\rho>=\frac{\hbar \Omega^{4}}{8 \pi^{2} c^{3}}
$$

The current wisdom is to argue that, if general relativity is valid up to the Planck scale, then one might guess that $\Omega$ is given by $\Omega_{p}$ where

$$
\hbar \Omega_{p}=E_{p}=\left(\frac{\hbar c^{5}}{G}\right)^{1 / 2},
$$

leading to a vacuum energy density, $<\rho_{p}>$ say, which is over a hundred orders of magnitude too large [2, 3].

Here, we argue that allowing $\Omega$ to have such a large value is completely ad hoc and not based on compelling physical arguments. Instead, we take the point of view that one should not consider vacuum fluctuations in isolation but rather in interaction with matter fields and, as a consequence, their contribution to the energy of the vacuum is much less. The fact is that all the well-known observed QED effects (Lamb shift, Casimer effect, etc.) involve interation of the vacuum field with matter. With that in mind, we prefer to use atomic units ( $\hbar=M=e=1$ [4], where $M$ and $e$ refer to the mass and charge of the electron, respectively). In these units, consistent with the fact that the atomic unit of velocity is $2.1877 \times 10^{8} \mathrm{~cm} / \mathrm{s}$, we note that $c=\alpha^{-1}=137.036$, where $\alpha$ is the fine-structure constant. Also, since $\left(G M^{2} / e^{2}\right)=2.401 \times 10^{-43}$, we see that in these units $G=2.401 \times 10^{-43}=$ $1.309 \alpha^{20}$. We note that $\alpha$ is the natural expansion parameter in QED. Thus, in atomic units (a.u.), (2) and (3) simply become 


$$
<\rho>=\frac{\alpha^{3}}{8 \pi^{2}} \Omega^{4}=4.92 \times 10^{-9} \Omega^{4}
$$

and

$$
\Omega_{p}=\alpha^{-5 / 2} G^{-1 / 2}(\text { a.u. })=0.874 \alpha^{-25 / 2}=4.49 \times 10^{26} .
$$

Thus, from (4) and (5), we obtain

$$
<\rho_{P}>=2 \times 10^{98} \text { a.u. }=1.2 \times 10^{96} \mathrm{~g} / \mathrm{cm}^{3} .
$$

This is to be compared with the measured vacuum energy density [1]

$$
<\rho_{v}>\approx 10^{-29} \mathrm{~g} / \mathrm{cm}^{3}=1.63 \times 10^{-27} \text { a.u. }
$$

In other words, $\left\langle\rho_{P}>\right.$ is too large by a factor of the order of $10^{125}$. This discrepancy is clearly unrealistically too large and we wish to explore how it may be reduced. In that context, we note that to get agreement between the theoretical value given in (4) and the observed value given in (7) requires an $\Omega$ value as low as $2.4 \times 10^{-5}$.

First, we recall an exact calculation which we carried out to obtain, within the framework of QED, the equation of motion of a radiating electron [5, 6]. Our analysis led to an explicit result for $\Omega$ given by

$$
\Omega=\frac{M-m}{M \tau_{e}}, \quad 0 \leq m \leq M,
$$

where $m$ is the bare mass, $M$ is the renormalized (physical) mass, and

$$
\tau_{e}=\frac{2 e^{2}}{3 M c^{3}}=\frac{2}{3} \alpha^{3} \text { a.u. }
$$

In addition, a striking feature of our calculation was the conclusion that causality considerations (which, in essence, state that effect follows cause and which lead to the technical fact that the poles of the electron response function must lie in the lower half-plane [7, 8]) dictate that $\Omega$ has a well-defined upper limit. We note that the limit $m=0$ corresponds to the largest cut-off value consistent with causality $\left(\Omega=\tau_{e}^{-1}\right)$. Thus, causality puts a restriction on the maximum value of $\Omega$. This choice had the virtue of corresponding to an electron of minimum size and it led to a simple second-order equation for the radiating electron, which 
was free of runaway solutions [6, 9]. However, with respect to how large $m$ is compared to $M$, as Feynman [10], among others, has noted it "- - cannot be determined theoretically". However, taking into account retardation and relativistic effects, most estimates [11] conclude that $m \approx(1-\alpha) M$. Substituting this result in (8) leads to the conclusion that the cut-off, $\Omega_{Q E D}$ say, is given by

$$
\Omega_{Q E D} \approx \alpha \tau_{e}^{-1}=\frac{3}{2} \alpha^{-2}=2.82 \times 10^{4}
$$

which is close to the value chosen by Bethe in his calculation of the Lamb shift. From (5), we see that this is smaller than $\Omega_{p}$ by a factor $\approx 1.72 \alpha^{21 / 2}=6.29 \times 10^{-23}$. Recalling, from (4), that $\left\langle\rho>\sim \Omega^{4}\right.$, we see that this choice reduces $\left\langle\rho>\right.$ by a factor of $8.75 \alpha^{42}=1.57 \times 10^{-89}$, to now give a vaccum energy density which is about $10^{36}$ times too large. How can this discrepancy be reduced further? Based on these numbers, one might expect that the energy contributed to the vacuum by the zero-point fluctuations occurs because of the interaction between these fluctuations and matter and not by consideration of these fluctuations in isolation. Clearly, more detailed work needs to be carried out but we feel that the reduction in the discrepancy between observations and theory which stem from the above considerations motivates more detailed analysis.

[1] M. Tegmark et al., Phys. Rev. D 69, 103501 (2004).

[2] S. Weinberg, Rev. Mod. Phys. 61, 1 (1989).

[3] P.J.E. Peebles, Rev. Mod. Phys. 75, 559 (2003).

[4] H.A. Bethe and E.E. Salpeter, "Quantum Mechanics of One- and Two- Electron Atoms" (Springer-Verlag, Berlin, 1957) pps. 2-3.

[5] G.W. Ford , J.T. Lewis and R.F. O’Connell, Phys. Rev. Lett. 55, 2273 (1985).

[6] G.W. Ford and R.F. O’Connell, Phys. Lett. A 157, 217 (1991).

[7] J.S. Toll, Phys. Rev. 104, 1760 (1956).

[8] R. Loudon, "The Quantum Theory of Light" (Clarendon Press, Oxford, 1973), p.67.

[9] J.D. Jackson, "Classical Electrodynamics", 3rd ed. (Wiley, New York, 1998), p.749.

[10] R.P. Feynman, "Quantum Electrodynamics" (Addison-Wesley, Reading, Mass., 1998), p.142. 
[11] J.J. Sakurai, "Advanced Quantum Mechanics" (Addison-Wesley, Reading, Mass., 1967), pps. 69 and 270-271. 